\title{
REVIEW \\ The contribution of neurophysiology in the diagnosis and management of cervical spondylotic myelopathy: a review
}

\author{
R Nardone ${ }^{1,2}$, Y Höller ${ }^{1}, \mathrm{~F}$ Brigo $^{2,3}$, VN Frey ${ }^{1,4}$, P Lochner $^{2}$, S Leis ${ }^{1}$, S Golaszewski ${ }^{1}$ and E Trinka ${ }^{1,4}$
}

Study design: Topical review of the literature.

Objective: The objective of this review article was to assess indications and usefulness of various neurophysiological techniques in diagnosis and management of cervical spondylogenic myelopathy (CSM).

Methods: The MEDLINE, accessed by Pubmed and EMBASE electronic databases, was searched using the medical subject headings: 'compressive myelopathy', 'cervical spondylotic myelopathy (CSM)', 'cervical spondylogenic myelopathy', 'motor evoked potentials (MEPs)', 'transcranial magnetic stimulation', 'somatosensory evoked potentials (SEPs)', 'electromyography (EMG)', 'nerve conduction studies (NCS)' and 'cutaneous silent period (CSP)'.

Results: SEPs and MEPs recording can usefully supplement clinical examination and neuroimaging findings in assessing the spinal cord injury level and severity. Segmental cervical cord dysfunction can be revealed by an abnormal spinal N13 response, whereas the P14 potential is a reliable marker of dorsal column impairment. MEPs may also help in the differential diagnosis between spinal cord compression and neurodegenerative disorders. SEPs and MEPs are also useful in follow-up evaluation of sensory and motor function during surgical treatment and rehabilitation. EMG and NCS improve the sensitivity of cervical radiculopathy detection and may help rule out peripheral nerve problems that can cause symptoms that are similar to those of CSM. CSP also shows a high sensitivity for detecting CSM.

Conclusion: Neuroimaging, especially magnetic resonance imaging, represents the procedure of choice for the diagnosis of CSM, but a correct interpretation of morphological findings can be achieved only if they are correlated with functional data. The studies reported in this review highlight the crucial role of the electrophysiological studies in diagnosis and management of CSM.

Spinal Cord (2016) 54, 756-766; doi:10.1038/sc.2016.82; published online 31 May 2016

\section{INTRODUCTION}

Cervical spondylosis (CS) is the most common cause of cervical myelopathy. ${ }^{1}$

The clinical sequelae of cervical spondylotic myelopathy (CSM) are a broad spectrum of motor and sensory abnormalities related to dysfunction of the cervical spinal cord. ${ }^{2}$ The diagnosis of CSM is essentially based on the combination of clinical signs/symptoms suggesting involvement of spinal long tracts (spastic paraparesis associated with a variable degree of lower limb ataxia) and clinical signs/symptoms indicating dysfunction of motor and sensory neurons in the cervical gray matter. ${ }^{3}$ However, sensory-motor deficits and reflex changes in the upper limbs can be missing; ${ }^{4}$ in these cases, CSM can be confused with other degenerative diseases such as amyotrophic lateral sclerosis (ALS). Magnetic resonance imaging (MRI) of the spinal cord can show signal abnormalities at the level of cord compression but gives no precise information on cervical cord dysfunction in CSM, as radiological data are discordant with clinical status in $50 \%$ of cases. ${ }^{5}$ Therefore, for the correct interpretation of the neuroimaging findings, the functional assessment of the long central pathways, as well as of motor and sensory neurones in the cervical gray matter, is helpful or even mandatory in patients with CSM.
We aimed in this review article to summarize the published articles dealing with electrophysiological studies in CSM patients, in order to assess their indication and usefulness. The MEDLINE, accessed by Pubmed (1966-April 2015) and EMBASE (1980-April 2015) electronic databases, was searched using the medical subject headings (MeSH): 'compressive myelopathy', 'cervical spondylotic myelopathy', 'cervical spondylogenic myelopathy', 'motor-evoked potentials', 'transcranial magnetic stimulation', 'somatosensory-evoked potentials', 'electromyograpy', 'nerve conduction studies' and 'cutaneous silent period'.

Two review authors (FB and PL) screened the titles and abstracts of the initially identified studies to determine whether they satisfied the selection criteria. Any disagreement was resolved through consensus. Full-text articles in English language were retrieved for the selected titles, and reference lists of the retrieved articles were searched for additional publications. In case of missing or incomplete data, principal investigators of included trials were contacted and additional information requested. The two reviewers independently assessed the methodological quality of each study and risk of bias, focusing on blinding and other potential sources of bias. The search strategy described above yielded 62 results. Only articles reporting data on

${ }^{1}$ Department of Neurology, Christian Doppler Klinik, Paracelsus Medical University, Salzburg, Austria; ${ }^{2}$ Department of Neurology, Franz Tappeiner Hospital, Merano, Italy; ${ }^{3}$ Department of Neurological, and Movement Sciences, Section of Clinical Neurology, University of Verona, Verona, Italy and ${ }^{4}$ Spinal Cord Injury and Tissue Regeneration Center, Paracelsus Medical University, Salzburg, Austria

Correspondence: Dr R Nardone, Department of Neurology, "F. Tappeiner" Hospital - Meran/o, Via Rossini, 5, Merano 39012 (BZ), Italy.

E-mail: raffaele.nardone@asbmeran-o.it

Received 2 June 2015; revised 24 March 2016; accepted 6 April 2016; published online 31 May 2016 
studies using the above-mentioned neurophysiological techniques in patients with CSM were considered eligible for inclusion; therefore, 58 papers were provisionally selected and contributed to this review: the earliest was published in 1979 and the most recent in 2015. A flow chart (Figure 1) illustrates the selection/inclusion process.

\section{APPLICATIONS AND FINDINGS}

\section{Electromyography and nerve conduction study}

Bilateral sensory disturbances in the hands should raise suspicion for cervical spinal cord pathology including CSM, and MRI of the same should be performed even if the electromyography (EMG)/nerve conduction studies (NCS) suggest bilateral carpal tunnel syndrome (CTS), when symptoms and/or clinical signs of cervical myeloradiculopathy are recognized. ${ }^{6}$ In this situation, the clinician needs to make a judgement as to whether the main problem is CSM or CTS and treat appropriately for severity and age.

Interestingly, both anatomical (segmental level and side) and pathophysiological (axonal type of lesion) requirements of the double-crush hypothesis are not supported by electrophysiological signs of median nerve mononeuropathy in most of the patients with concomitant CSM and carpal tunnel syndrome. In fact, Bednarik et al. ${ }^{7}$ demonstrated a statistically significant association between CSM and carpal tunnel syndrome but failed to find any evidence of etiological relationship between these two conditions.
In a recent study, the value of preoperative MRI combined with EMG for predicting functional outcome following surgical management of CSM has been evaluated. ${ }^{8}$ Ninety-four patients were classified into four groups on the basis of the presence $(+)$ or absence $(-)$ of increased signal intensity on T2-weighted MRI and on the positive $(+)$ /negative $(-)$ results of the EMG. Four types were thus identified: type I, MRI/EMG (-/-); type II, MRI/EMG (+/-); type III, MRI/EMG $(-/+)$; and type IV, MRI/EMG $(+/+)$.

A significant difference in the recovery ratio between the four study groups was found. The clinical outcome after surgery was best in type I patients and worst in type IV patients. Patients who had a negative EMG and those without an increased signal intensity on T2-weighted MRI only suffered from mild symptoms: they had a short disease duration and, importantly, experienced a good surgical outcome.

CS, isolated or combined with CSM, may coexist with ALS and other motor neuron diseases (MND). It is important to detect ALS coexisting with CS at an early stage, especially when surgical treatment is planned.

A single-fiber EMG study has been performed in 26 patients with ALS, 19 with ALS and CS and 22 with isolated CS.9 ${ }^{9}$ Mean jitter, percentage of jitter $>55 \mu$ s and percentage of impulse blocking were not statistically different between ALS patients with and without concomitant CS but were significantly lower in patients with isolated CS. Mean fiber density was not statistically different among the three patient groups. The percentage of pairs with a jitter $>55 \mu$ s exceeding
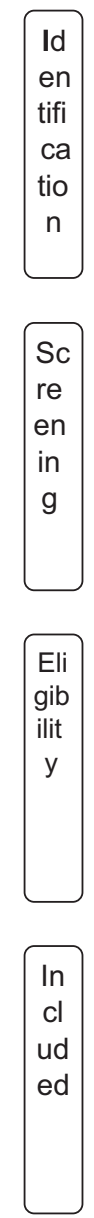

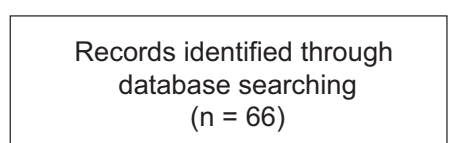

$$
(n=66)
$$
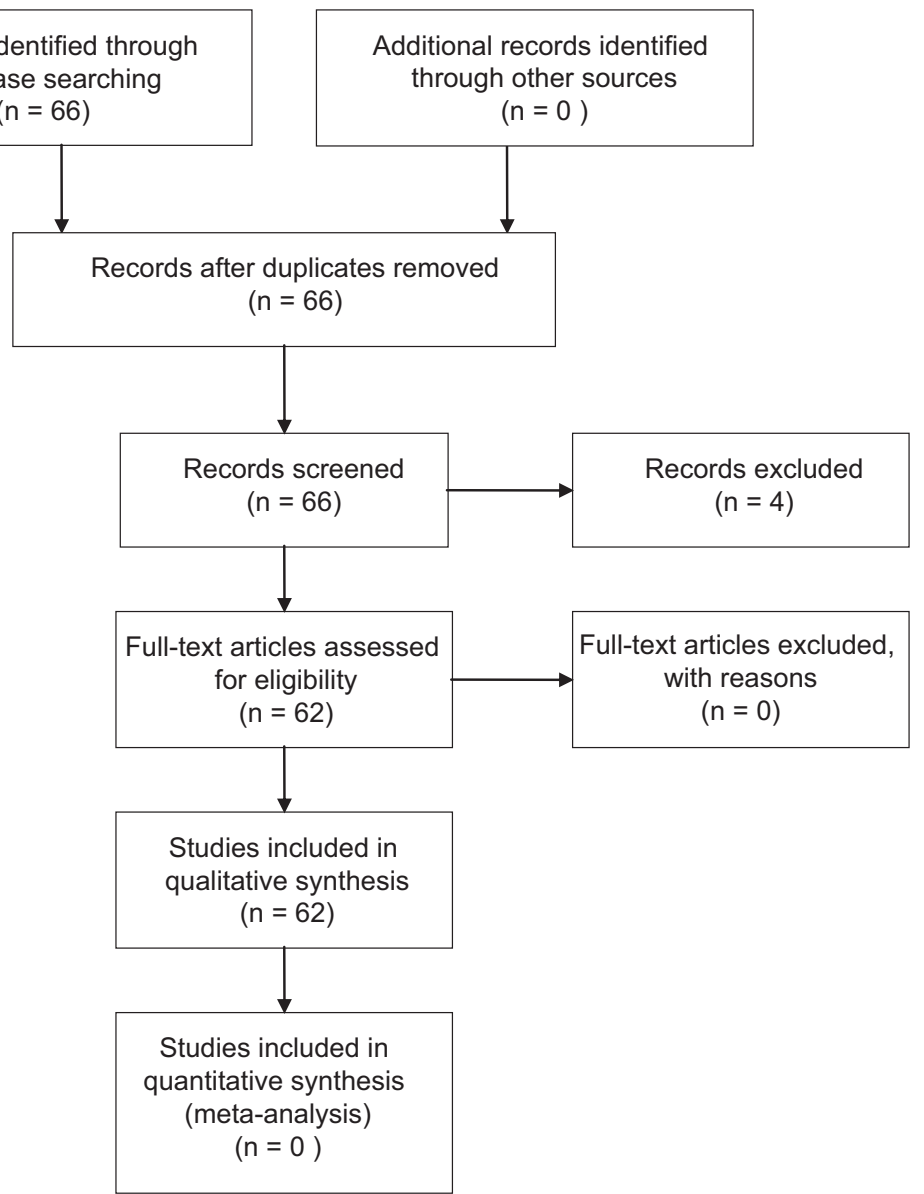

Figure 1 Flow chart illustrating the selection/inclusion process. 
$40 \%$ and mean jitter exceeding $55 \mu$ s had a high sensitivity and specificity in the diagnosis of ALS.

The authors concluded that single-fiber EMG can provide valuable information in differentiating ALS from radiculopathies due to CS. However, they failed to explain why single-fiber EMG, a test of the neuromuscular junctions, should be more frequently affected in ALS (or lower MND). On the other hand, ALS/MND is a clinical diagnosis supported by clinical neurophysiology criteria that do not include single-fiber EMG (not recommended by International Federation of Clinical Neurophysiology (IFCN)) and extend beyond testing the cervical segment.

By means of surface EMG also the contributions of individual muscles to a movement pattern can be examined. Compared with healthy subjects, patients with CSM have a prolonged duration of activation of biceps femoris and tibialis anterior, prolonged co-activation of rectus femoris and biceps femoris, and impaired scaling of the amplitude of rectus femoris and biceps femoris (Figure 2). ${ }^{10}$ The timing parameters of rectus femoris, tibialis anterior and medial head of gastrocnemius were considered sufficiently reliable for use in clinical practice, whereas the reliability of biceps femoris is questionable. The prolonged activation of tibialis anterior was not accompanied by co-activation with medial head of gastrocnemius. Its isolated prolonged activation may have been a strategy to increase the stability of the ankle during stance due to impaired proprioception and joint position sense, which has been been identified in CSM, whereas analysis of muscle responses to lengthening suggested that spasticity did not contribute significantly to this finding. Therefore, EMG provides insight into the underlying impairments that characterize an abnormal gait. These results provide evidence that paresis is an important contributory factor to gait impairment in CSM, as indicated by impaired amplitude and the need for proximal co-activation to compensate for lack of distal power generation. These findings may have relevant implications for the assessment of gait impairment in CSM and may also lead to the development of new rehabilitation strategies.

Routine NCS did not evaluate adequately spinal cord function after compression lesions. On the contrary, combined utilization of multiple F-wave parameters (minimal F latency, $\mathrm{F}$ chronodispersion, $\mathrm{F}$ persistence and side-to-side differences) resulted in 55\% sensitivity and $100 \%$ side concordance for detecting radiculopathy in CS, as considered in comparison with MRI. ${ }^{11}$ This method can thus be considered a useful diagnostic adjunct in the electrophysiological evaluation of degenerative cervical spine conditions.

Lo and colleagues first demonstrated that cutaneous silent period (CSP) measurements are simple, quick and sensitive methods for the diagnosis of CSM (Figure 3). ${ }^{12}$ Successively, Stetkarova and Kofler ${ }^{13}$ aimed to assess the utility of CSP for functional evaluation of mild CSM. They found that CSP onset and end latencies were delayed and that CSP duration was shortened in patients with CSM. The authors concluded that CSP abnormalities were almost equally sensitive as upper limb MEPs and were highly associated with spinothalamic dysfunction. This finding also suggests a high correlation of CSP abnormalities with corticospinal tract dysfunction and a supraspinal influence on CSPs. However, the use of CSP in clinical practice is rather limited, as this technique is particularly useful in evaluation of certain segments of the nervous system or of certain components of sensory nerves that are not well assessed by other routine electrodiagnostic methods.

Sternocleidomastoid muscle EMG and dermatomal somatosensory-evoked potential (dSEP) were evaluated as diagnostic aids in differentiation between ALS and CSM in another interesting study. ${ }^{14}$
Both sternocleidomastoid muscle-EMG and dSEP have proved to be valuable differential aids between these two pathologic conditions.

\section{Somatosensory-evoked potentials}

SEPs can be used to disclose abnormalities of ascending sensory pathways in CSM. A number of studies that utilized cephalic reference montages showed that dorsal column dysfunction can be demonstrated in $43-100 \%$ of patients by lower limb SEPs, in $57-74 \%$ of patients by ulnar nerve SEPs and in $24-59 \%$ of patients by median nerve SEPs. ${ }^{15-18}$

In these studies, upper limb SEPs rarely showed abnormalities in patients without sensory deficits. On the other hand, abnormal lower limb SEPs are of no value for localizing the dysfunction at the cervical level. The non-cephalic reference (shoulder contralateral to stimulating site) recordings of SEPs allow a separate analysis of the dorsal horn N13 response and of the P14 potential, the latency of which is thought to reflect the transit time of the ascending volley up to the lower brainstem level. Abnormalities of the N13 potential have been found in diseases affecting the central gray matter, such as syringomyelia or intramedullary tumors. ${ }^{19}$ An abnormal spinal N13 potential was found in $95 \%$ of radial, $90 \%$ of median and $54 \%$ of ulnar nerve SEPs in 11 patients with MRI-confirmed CSM. ${ }^{20}$ In contrast, scalp SEPs reflecting the activity of the dorsal column system up to the parietal cortex were normal in the vast majority of the CSM patients. Therefore, segmental cervical cord dysfunction in CSM is principally manifested by an abnormal spinal N13, whereas scalp far-field abnormalities are associated with dorsal column involvement. ${ }^{21}$

In a successive report, Restuccia et al. ${ }^{22}$ addressed the question of whether the diagnostic yield of SEPs in CSM can be improved by assessing separately dorsal column and dorsal horn responses to stimulation of median, radial and ulnar nerves. This study shows that the recording of both spinal and scalp SEPs in patients with CSM can reveal two main types of cord dysfunction at the cervical level. The most frequent SEP abnormality was the absence or reduction of the spinal N13 potential, which was evident at least for one of the tested nerves in all but two patients, in whom the spinal cord compression was located at the $\mathrm{C} 2-\mathrm{C} 3$ levels.

The N13 abnormality was often associated with normal scalp SEPs, as the loss of N13 with normal P14 and N20 potentials has been found in 28 of 76 upper limbs. Therefore, an isolated abnormality of the N13 potential in patients with CSM is likely to reflect an anatomical damage limited to the cervical gray matter. In fact, a loss of N13 with normal P14 and N20 potentials has been reported in lesions of the cervical gray matter leaving dorsal columns unaffected. ${ }^{23-27}$ The N13 abnormality in patients with CSM is likely to reflect a decreased blood supply to cervical cord from anterior spinal artery. ${ }^{20,21}$

By contrast, in earlier studies, a neck-to-forehead montage for the recording of the N13 potential was used, and the detection rate of upper limb SEPs abnormalities was much lower. ${ }^{28}$ In fact, earlier SEP studies in patients with CSM failed to reveal any selective loss of the N13 potential in association with normal scalp potentials. The cervical response recorded with a neck-to-forehead montage, which is an amalgam of the cervical N13 and scalp P14 potentials, ${ }^{29,30}$ is not significantly affected by selective lesions of the cervical gray matter. ${ }^{26,26}$

Another reason for the high rate of $\mathrm{N} 13$ abnormalities in the patients studied by Restuccia et al. ${ }^{22}$ pertains to the use of multiple nerve stimulation, including the radial nerve, which corresponds to the more frequently involved level. Interestingly, abnormalities of the N13 potential are strongly related to the compression level as revealed by radiological investigations. 

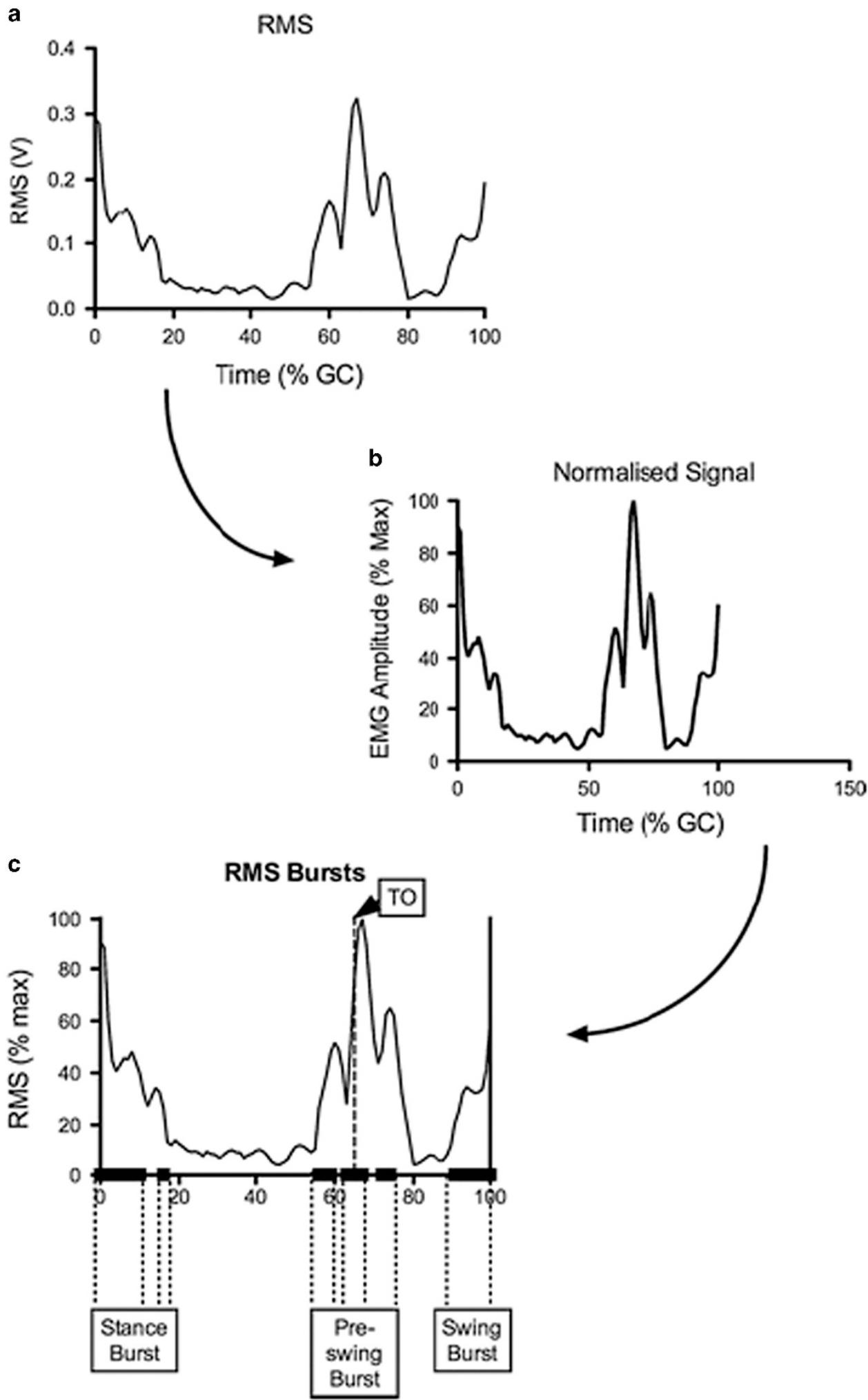

Figure 2 Root mean-square amplitude of rectus femoris muscle over gait cycle from one participant with CSM (a), normalization (b) and extraction (c) of amplitude over bursts of muscle activation. GC, gait cycle; RMS, root mean square; TO, toe off; V, volts; \% max, percentage of maximum RMS amplitude. Reproduced with permission from Malone et al. ${ }^{10}$

Indeed, the higher rate of reduced N13 potentials in radial and median nerve SEPs than in ulnar nerve SEPs (93.5 and $84.6 \%$ vs $65.3 \%)$ correlates well with the involvement of the C5 level as shown by MRI.
Another consistent SEP feature in the CSM patients evaluated with the non-cephalic reference montage was the abnormality of the P14 potential, which was found in $37.6 \%$ of radial, in $41 \%$ of median and in $62.8 \%$ of ulnar scalp SEPs. ${ }^{22}$ The P14 scalp far-field potential is 


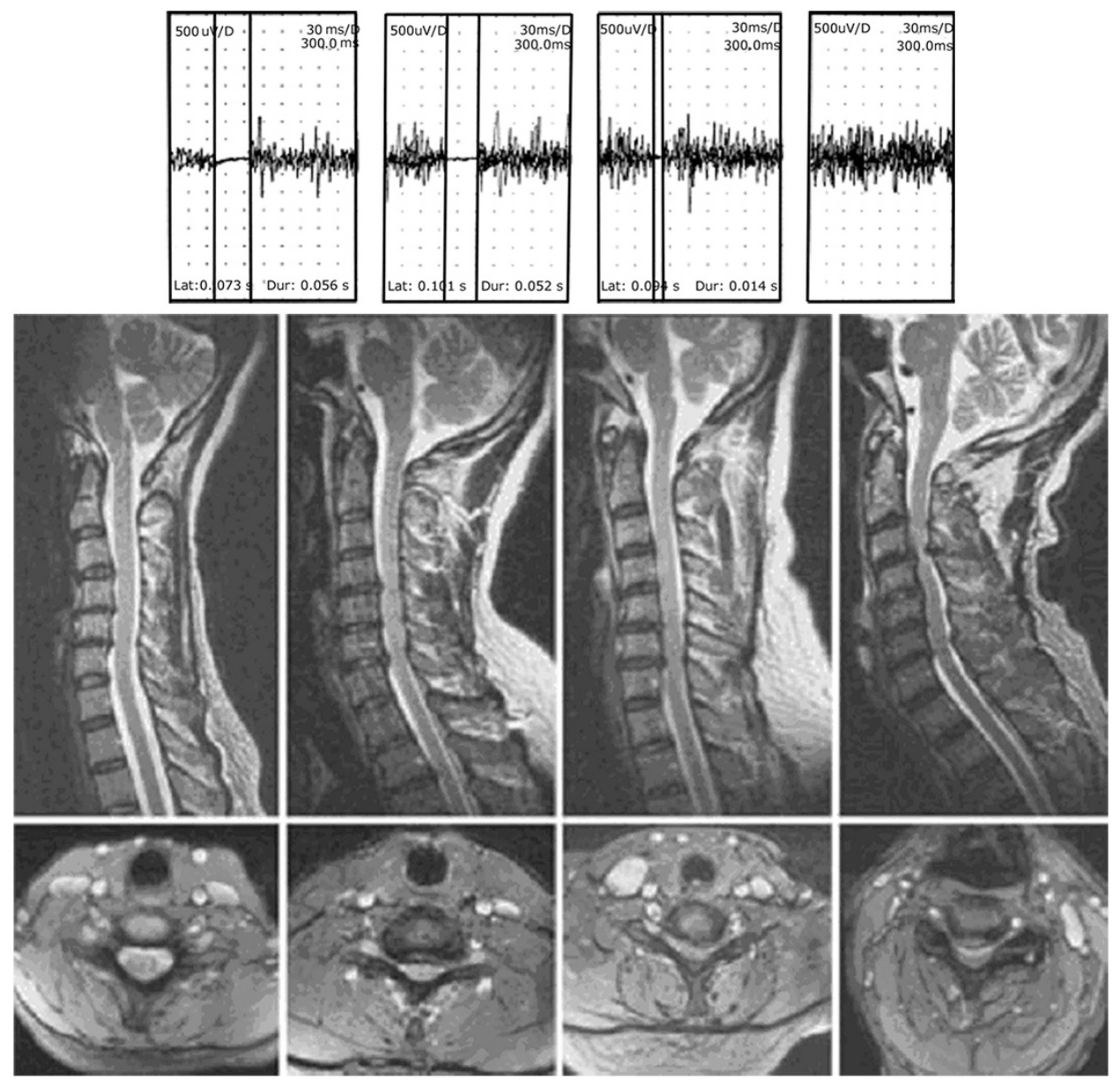

Figure 3 Sagittal turbo spin echo T2-weighted (top) and axial gradient echo magnetic resonance images of the cervical spine of Cases 22, 20, 4 and 6 showing increasing severity of cord compromise, depicted as Groups 1 to 4, respectively. Actual cutaneous silent period (CSP) tracings of Cases 22 (normal CSPs), 20, 4 and 6, respectively. Stimulation of right $V$ digit was performed with abductor pollicis brevis recordings. Superimposition of three traces is shown. The CSP latency, duration, vertical gain and horizontal sweep speed are depicted in tracings. No CSP was elicited for Case 6. Reproduced with permission from Lo et al. ${ }^{12}$

generated in the brainstem tracts of the ascending lemniscal pathways close to the cervicomedullary junction. ${ }^{28,30}$ The increased P9-P14 interval or absent P14 with a normal P9 thus directly reflects time dispersion at the level of the cervical spinal cord.

In earlier studies, which used a cephalic reference montage, scalp SEP abnormalities in CSM were frequently represented by the abolition or latency delay of the parietal N20 response. However, prolonged N20 latency or N13-N20 interval provides an indirect assessment of abnormal dorsal column function and includes the transit times in medial lemniscus and thalamocortical fibers. Therefore, an N20 abnormality can be caused by any intracranial conduction slowing in the somatosensory pathways. The evaluation of the scalp P14 far-field provides more reliable information on dorsal column dysfunction than that of the parietal N20 potential. For this reason, the percentage of patients with subclinical dorsal column dysfunctions revealed by upper limb SEPs (normal joint and touch sensation but abnormal scalp SEPs) was greater in the series of Restuccia et al. ${ }^{22}$ than in those reported in earlier studies. ${ }^{17,18}$ The resynchronization of the ascending volley in the intracranial somatosensory pathways may account for the abolition or the latency delay of the P14 potential with a normal N20 latency. ${ }^{31}$ With respect to the relationship between scalp SEP abnormalities and compression level, ulnar scalp SEPs are more often involved than median scalp SEPs. ${ }^{17,18}$ A greater sensitivity of the longer fibers to compression could explain the higher rate of abnormal ulnar nerve SEPs compared with median nerve SEPs in CSM. ${ }^{22}$ However, because of the small difference in length between the fibers incoming from median and ulnar nerves, this hypothesis seems unlikely, even though it might hold for the high detection rate of tibial nerve SEPs in CSM.

In another study, abnormal patterns of the SEPs were compared with the abnormal-evoked spinal cord responses recorded, in 13 patients with various cervical lesions, from the posterior epidural space using catheter electrodes or needle electrodes inserted into the ligamentum flavum. ${ }^{32}$ Cervical N13 potentials were recorded by two different montages. Lower cervical N13 (LC-N13) recorded from the Cv5-anterior neck montage showed similar latency to upper cervical N13 (UC-N13) recorded from the Cv2-inion montage. Attenuation of the LC-N13 and relatively preserved UC-N13 and P13-P14 were 
a

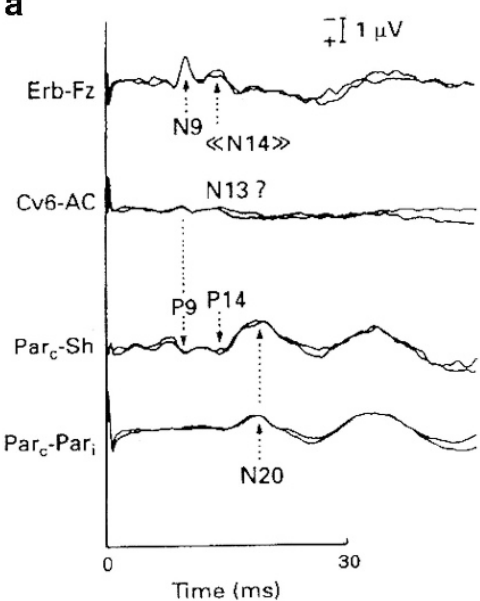

b

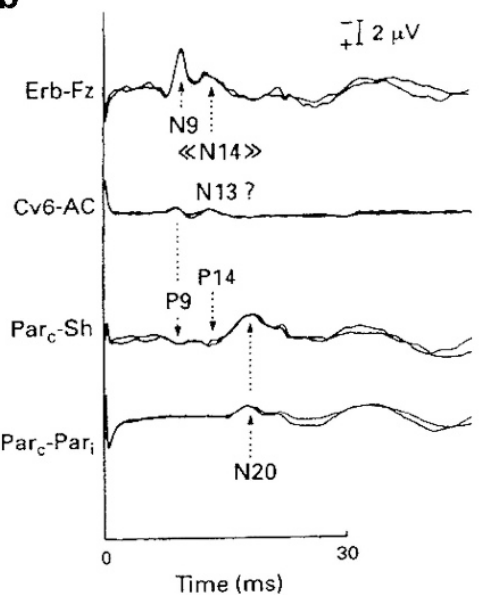

C

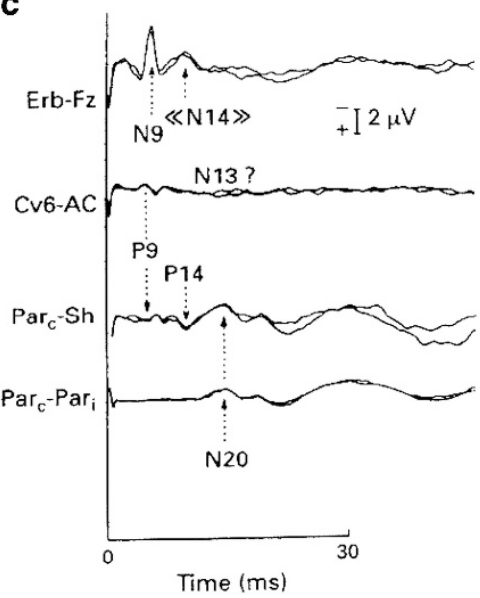

Figure 4 Abnormal spinal and scalp SEPs in a patient with CSM. Right radial (a), median (b) and ulnar nerve SEPs. The peak latencies of N9 and P9 responses were within normal limits. The N13 potential was absent after stimulation of the radial (c) and median nerves but normal after stimulation of the ulnar nerve. The peak latencies of the P1 response, as well as the P9-P14 intervals, were delayed after stimulation of the radial ad median nerves and not evaluable after stimulation of the ulnar nerve. Reproduced with permission from Restuccia et al. ${ }^{22}$

characteristic in patients with cervical syringomyelia and CSM at the mid-cervical levels, whereas attenuation of the UC-N13 with normal LC-N13 was characteristic in patients with CSM who showed conduction blockade of the evoked spinal cord responses at the C3-4 level. Therefore, two different cervical N13 potentials can be recorded by two different montages, and they represent different behavior in different spinal cord lesions. In this study, the authors were able to demonstrate that at least the early part of the P13-P14 complex originates in the upper cervical region.

Furthermore, valuable information can be provided by dSEPs in selected patients with multiple root or spinal cord involvement. ${ }^{33}$ In two patients with lesions causing multiple root involvement, dSEPs helped reveal the functional abnormality. In a patient with an MRI-verified lesion occupying a considerable volume of the lower spinal cord, all neurophysiological investigations were normal (EMG, MEPs, median and tibial SEPs), except for the dSEPs; abnormal dSEPs were also found in patients with a small well-circumscribed intramedullary lesion at right C2 level.

\section{Motor-evoked potentials}

Many studies have employed MEPs elicited by transcranial magnetic stimulation (TMS) in patients with CSM to assess the central motor pathways. They have demonstrated any abnormalitiy for distal upper limb muscles and for lower limb muscles in almost all patients. ${ }^{34-45}$ The MEP abnormality consists usually of a prolonged central motor conduction time (CMCT), but one study showed that, in addition to the CMCT, the duration of MEPs may be helpful in evaluating patients with CSM. ${ }^{46}$

For careful assessment of the cervical spinal cord function, MEPs should be recorded from multiple upper limb muscles corresponding to different segmental levels, in particular from the biceps and thenar muscles to study the central motor pathways of two different myotomes, C5-C6 and C8-D1. ${ }^{36}$ In patients with a high degree of cervical cord compression, the CMCT is prolonged for the biceps brachii and distal hand muscles. Conversely, in patients with multilevel cervical cord compression such as cervical canal stenosis, CMCT was found to be abnormal for the thenar muscle and normal for the biceps muscle. ${ }^{36}$ This finding suggests a prevalent damage of the central motor pathways for distal upper limb muscles. Also, in patients who have a single compression level, such as a central disc herniation, the CMCT abnormality is usually confined to distal upper limb muscles.

When the patients show a close correlation between MRI and MEP findings, the most important etiological factor is probably a segmental demyelination of central motor pathways due to a direct mechanical spinal cord compression. However, more than one single mechanism may have a role in the pathogenesis of spinal cord damage, and sometimes discrepancies can be observed between the level of spinal cord compression documented by neuroimaging studies and the level of spinal cord dysfunction revealed by MEPs. A more caudal functional involvement of the cervical cord revealed by MEPs can be explained on the basis of a compromised blood flow to the spinal cord. The anterolateral regions of lower cervical segments are blood supplied almost exclusively from the anterior spinal artery, whereas the higher cervical segments that are located between the cervical and intracranial arterial territories have more sources of blood. Therefore, the frequent involvement of lower cervical segments probably depends on their higher vulnerability to ischemic damage. When cervical spondylotic compression involves the anterior spinal artery, the major damage is of vascular origin and localized to lower cervical segments, independent from the level of spondylotic degenerative changes. ${ }^{47}$

It should be considered that similar MEP findings can be observed in a wide array of neurological disorders such as neoplastic compression, intraspinal tumors, myelitis, spinal forms of multiple sclerosis and MND.

MEP studies may reveal a subclinical involvement of corticospinal projections to myotomes that are innervated by nerve roots of the cervical spinal cord. ${ }^{36}$

In some patients (approximately 15\% of CSM cases), who present with an apparently isolated spastic paraparesis, CMCT is normal for proximal upper limb biceps muscles but abnormal for distal upper limb muscles and for all muscles supplied by more caudal myelomeres. When this pattern of MEP abnormality is observed, MRI examination of the cervical spinal cord should necessarily be performed. On the contrary, an abnormal central motor conduction for the biceps muscle may indicate several other conditions. In fact, this finding can be recorded in a high cervical cord compression or in 
intracranial disorders involving central motor pathways, MND, as well as in a neurodegenerative disorder involving the spinal cord.

The examination of central conduction for more cranial myotomes, and in particular the MEPs of the trapezius muscle, may also be of great importance. In a study aiming at assessing whether trapezius MEPs are helpful in the evaluation of high cervical cord lesions, $43 \%$ of the patients with radiological evidence of corticospinal lesion above the level of C5 and 14\% without this radiological evidence showed prolonged MEP latencies. ${ }^{37}$

The differential diagnosis between an intracranial disorder and a high cervical cord lesion may be clinically and electrophysiologically difficult. The technique of cervicomedullary junction stimulation enables the selective evaluation of the intracranial and the spinal segments of central motor pathways to the proximal upper limb muscles, by subtracting the latency of the MEPs evoked by paravertebral magnetic stimulation from the latency of the MEPs evoked by cervicomedullary junction stimulation. ${ }^{48,49}$

In addition to the localization of lesion sites, MEPs may provide valuable information in quantifying the degree of functional involvement of the spinal cord, in particular when MRI shows only a minor spondylogenic compression of the spinal cord, which can have no significant functional effect on the spinal cord. In a combined MEP and MRI study, the level of spinal cord compression was determined according to the pattern of CMCT prolongation and compared with the level disclosed by MRI (Figure 4)..$^{50}$ The actual level of compression was correctly indicated by TMS in $87.5 \%$ of cases and by MRI in $12.5 \%$ of cases. MEP recording may help in the selection of patients with severe dysfunction of central motor pathways who are probably better candidates for surgery. In particular, serial MEP recording allows identifying the progressive forms of CSM and in selecting patients who may benefit from surgical therapy. MEPs may be helpful in determining the surgical site in patients who have multilevel disc herniations and can also have a role in monitoring the effect of operative intervention.

In the most recent TMS study, the sensitivity of the CMCT recorded from the abductor digiti minimi was $92 \%$ for C3/4 myelopathy, $95 \%$ for $\mathrm{C} 4 / 5,58 \%$ for $\mathrm{C} 5 / 6$ and $9 \%$ for $\mathrm{C} 6 / 7 .{ }^{51}$ Therefore, CMCT recorded from abductor digiti minimi is useful for the screening of sternocleidomastoid muscle rostral to the C5/6 level, whereas the diagnosis of patients with $\mathrm{C} 6 / 7$ sternocleidomastoid muscle should include assessment of the MEPs recorded from abductor hallucis. Patients with C6/7 myelopathy may lack clinical symptoms in their hands and CMCT to abductor digiti minimi tended to be less prolonged, while they may only show symptoms in their lower limbs as gait disturbance. ${ }^{52}$

\section{ELECTROPHYSIOLOGY AND CSM SURGERY}

\section{Preoperative assessment}

The functional electrophysiological evaluation obtained pre-surgically by evoked potentials is useful for outcome prediction after surgical treatment of CSM. Evoked potentials have been correlated with clinical findings, both pre- and postoperatively. In an MEP study, De Mattei et al. ${ }^{45}$ first demonstrated a significant CMCT improvement in 11 of 12 patients with cervical myelopathy of varied etiology who underwent decompressive surgery. Similar findings have been reported in another study, but the preoperative MEPs were found not to provide predictive information for clinical outcome. ${ }^{53}$ Neurological improvement was accompanied by increased thoracolumbar spinal cord CMCT after decompressive surgery only in patients with mild $\mathrm{CSM} ;{ }^{54}$ no electrophysiological parameter has been identified in this study as an independent preoperative predictor.
In a retrospective study, MEPs were used for the assessment of spinal cord function before and after surgery, and MEP changes correlated with clinical findings. ${ }^{55}$ Early surgical intervention for CSM was found to produce a beneficial effect on spinal cord functionality that was revealed by MEP studies.

Interestingly, sufficient clinical results of surgical treatment can be expected even in CSM patients who are older than 80 years of age, when the patients are correctly selected by assessment with MEPs. ${ }^{56}$

Important preoperative issues can also be provided by SEP studies. Preoperative recording of the N9-N20 interval of the median SEP has been reported to predict good functional outcome in 39 patients operated for severe CSM. ${ }^{57}$ Moreover, isolated N13 abnormalities also predicted favorable surgical outcome in two studies comprising 49 and 11 CSM patients, respectively. ${ }^{58,59}$ Finally, the findings of a recent study indicate that, in comparison with conventional averaging SEP, the latency variability of trial-to-trial SEP recorded preoperatively presented much higher sensitivity and specificity in predicting surgical outcomes of CSM. ${ }^{60}$ In fact, analysis of single-trial signals, rather than the across-trial ensemble averaging signals, can better detect changes in SEP. ${ }^{61}$

\section{Intraoperative monitoring}

Intraoperative electrophysiological monitoring has an important role in high-risk spinal cord injury. Initially, spinal cord-evoked potentials were recorded by using electrodes inserted into cervical intervertebral discs after caudal epidural stimulation. ${ }^{62,63}$ This method enables localization of the conduction block at the myelopathic spinal cord level intraoperatively but is relatively invasive. More recently, recording epidural spinal cord-evoked potential during surgery allows a more accurate functional localization and even a correlation with functional outcome. ${ }^{64}$

SEPs provide an efficacious method to objective evaluate the functional integrity of the spinal cord. ${ }^{65}$ SEPs are non-invasive, technically easy and simple to obtain, and relatively free from interference of inhalated anesthetic agents. An important limitation of this technique is that obviously the anterior motor pathways are not explored. Moreover, in a study comparing 577 patients with intraoperative SEP monitoring and 462 patients, ${ }^{66}$ one patient in the operative group was found to develop a partial central cord syndrome despite having unobtrusive intraoperative SEP values.

These shortcomings can be addressed by employing MEPs. MEPs can be recorded from the upper and lower extremities or via transcutaneous epidural electrodes. ${ }^{67}$

Satisfactory results for eliciting MEPs during everyday practice can be achieved with constant-voltage stimulators. ${ }^{68} \mathrm{~A}$ short train of stimuli is preferable when eliciting muscle MEPs in anesthetized patients. This generates multiple descending volleys that are carried by the fast corticospinal tract neurons and cause the final depolarization of the spinal cord's alpha-motoneurons. The most efficient duration of the stimulus in the train is $0.5 \mathrm{~ms}$; the optimal interstimulus interval, regardless of intensity, is $4 \mathrm{~ms}$; a train repetition rate of $2 \mathrm{~Hz}$ is suggested as optimal. ${ }^{68}$

It should be noted that cortical MEPs elicited by TMS are particularly sensitive to interference by certain halogenated volatile anesthetic agents. However, the administration of total intravenous anesthesia maintained with a target controlled infusion enables successful SEP and MEP monitoring. ${ }^{69,70}$ Lo and colleagues ${ }^{71}$ demonstrated the use of desflurane for successful intraoperative acquisition of MEPs as an alternative anesthetic regime in subjects who underwent scoliosis surgery. In a successive study, the same research group compared the use of desflurane and total intravenous 
anesthetic regimens showing that both anesthetic regimens allowed successful monitoring MEPs during spinal surgery. ${ }^{59}$

A large study of 427 patients provided evidence that MEPs were of high sensitivity for identifying motor tract injury during anterior and posterior cervical decompression. ${ }^{72}$ Surgeons should therefore strongly consider using MEPS when operating on patients with CSM, in particular on those with ossification of the posterior longitudinal ligament.

Besides CMCT abnormalities, also a significant correlation between intraoperative decreased MEP amplitudes and postoperative new neurological deficits has been reported in patients with CSM. ${ }^{73}$ Nevertheless, the development of postoperative segmental motor paralysis after cervical laminoplasty for CSM was found to occur even if there were no abnormal findings during intraoperative monitoring. ${ }^{74}$ Moreover, in a retrospective review among 1445 patients who underwent anterior cervical surgery, ${ }^{75} 18.4 \%$ have significant neurophysiological alerts during the procedure, but none of them showed sustained postoperative neurological deficits.

During cervical laminectomy, especially patients with CSM at the C4-C5 level are susceptible to C5 injury. ${ }^{76}$ However, two studies that simultaneously utilized intraoperative MEPs and EMG minimized the occurrence of this complication. ${ }^{77,78}$

Fan et al. ${ }^{77}$ conducted a retrospective study for 132 patients, who were monitored with conventional techniques including ulnar and posterior tibial nerve SEPs, C5-C7 dSEPs and transcranial electrical stimulation (TES)-induced MEPs recorded from hand and leg muscles. Moreover, prospective studies were applied to other 68 patients, for whom TES-induced MEPs and spontaneous EMG were monitored from deltoid and biceps muscles in the awake state. In the retrospective cohort, six patients presented after surgery with C5 nerve root palsy showing unilateral deltoid muscle paralysis despite entirely unremarkable conventional SEPs, dSEPs and TES-induced MEPs from upper and lower limbs. In the prospective cohort, two patients experienced C5 motor nerve root palsy. ${ }^{77}$ Impending C5 nerve root injuries after laminectomy were successfully identified in both patients by detecting significant changes in deltoid and biceps TES-induced MEPs and spontaneous EMG, thereby averting more serious consequence.

In another retrospective study, ${ }^{78}$ in 238 subject who underwent anterior cervical spinal procedures, spinal nerve root function was monitored by means of TES-induced MEPs and spontaneous EMG activity. The two techniques provide complementary information, but MEPs show increased sensitivity to C5 deficit compared with spontaneous EMG activity alone, because of the risk of false-negative findings obtained using only EMG activity.

Finally, a systematic review aiming to use evidence-based medicine to examine the diagnostic and therapeutic utility of intraoperative electrophysiological monitoring in the surgical treatment of cervical degenerative disease should be reported. ${ }^{79}$ The authors of this review concluded that, although the use of MEP/SEP monitoring may serve as a sensitive means to diagnose potential neurological injury during anterior spinal surgery for CSM, intraoperative MEP/SEP worsening is not specific; it may not represent clinical worsening and its recognition does not necessarily prevent neurological injury, nor does it result in improved outcome (Class II). Moreover, intraoperative improvement in MEP/SEP parameters/indices does not appear to forecast outcome with reliability (conflicting Class I data).

\section{DISCUSSION}

CSM is the most frequently observed myelopathy. The first step in the diagnosis of CSM and other spinal cord disorders should be history taking and clinical examination. However, in subjects with CSM, physical examination findings are not always consistent with severity of disease, and the use of neuroradiological examinations is essential to establish the correct diagnosis. In particular, radiologic evidence of spondylotic cord damage is necessary for the diagnosis of CSM. MRI is usually the study of choice and can demonstrate or exclude spinal cord compression in these patients.

On the other hand, neuroimaging is not always completely accurate for making a diagnosis, and the functional involvement of the spinal cord cannot be assessed by MRI. The neurophysiological evaluation by means of different types of electrodiagnostic studies may thus be considered the necessary 'trait d' union' between clinical assessment and radiological investigations. ${ }^{47,80}$

EMG and NCS, as well as SEPs and MEPs elicited by means of TMS, have an important role in differentiating CSM and nerve root compression from peripheral neuropathies and myopathies. These currently available electrophysiological methods may disclose segmental dysfunction of the anterior horns (EMG) and of the posterior horns (SEP), as well as of the long spinal pathways (SEP and MEP), and should be highly recommended in patients with MRI evidence of symptomatic or asymptomatic spinal cord compression.

EMG may reveal muscular denervation in cases in which CSM and radiculopathy have been present for a prolonged period of time. Differentiation between EMG signs of motor radicular damage and the involvement of anterior horn cells is difficult, somewhat arbitrary and there exists inevitably at least a partial overlap between these conditions.

NCSs are usually within the normal limits in CSM and spondylogenic radiculopathies (except in the cases with extensive axonal or upper motoneuron damage) because the distal part of the peripheral nerve is normal in these conditions. In contrast, NCSs frequently show slowed conduction in subjects with peripheral nerve entrapment or in many peripheral neuropathies.

Objectifying the presence and degree of sensory and/or motor involvement in patients with CSM may be helpful for establishing the diagnosis and in therapeutic decision-making.

SEPs are able to detect even subclinical abnormalities of central sensory pathways. In particular, an usual finding is the isolated loss of the spinal N13, and the recording of the cervical N13 response can be considered a reliable diagnostic tool in patients with CSM, whereas the less frequent abnormalities of the scalp far-field P14 is very useful in disclosing dorsal column dysfunction.

Furthermore, a very high sensitivity of the MEPs elicited by TMS has been demonstrated in spinal cord disorders, including CSM. ${ }^{81}$ MEPs can help in localizing the main site of interest for later neuroradiological studies or in detecting a subclinical involvement of central motor pathways. Extremely helpful is the multilevel assessment of the CMCT, which can provide functional correlate to radiological abnormalities. The functional assessment of corticospinal conduction by means of MEP can thus significantly contribute to the diagnosis and management of CSM. Especially the differential diagnosis between CSM and MND may be difficult because both conditions are characterized by a variable combination of upper and lower motoneuron signs. In subjects with spinal cord compression, CMCT abnormalities are usually recorded in all muscles localized below the level of the spinal lesion, whereas the muscles supplied by more cranial myelomeres are spared. In MND, abnormal MEP findings recorded from certain muscles may be associated with a normal CMCT to muscles innervated by more caudal myelomeres, thus leading to a peculiar 'suspended' abnormality of the central motor conduction. Typically, in patients with CSM, an abnormal CMCT 
for the biceps brachii may be associated with normal MEP findings for distal upper and lower limb muscles. ${ }^{36}$

This suspended abnormality of central motor conduction for biceps brachii could also be determined by a delay of conduction along the C5 and C6 roots. A concomitant lesion of these roots should be excluded by clinical examination and/or by EMG study of muscles innervated by $\mathrm{C} 5$ and $\mathrm{C} 6$ roots. A CMCT abnormality for proximal upper limb muscles with normal conduction to distal upper limb muscles can rarely be observed in patients with CSM. ${ }^{36}$ A suspended central conduction abnormality can also be characterized by an abnormal CMCT for distal upper limb muscles associated with a normal conduction for lower limb muscles. In these cases, the evaluation of the central motor conduction with the F-wave method is very useful or even mandatory for a correct localization of motor pathway dysfunction. Moreover, combined evaluation of multiple F-waves parameters (minimal F latency, $\mathrm{F}$ chronodispersion, $\mathrm{F}$ persistence and side-to-side differences) resulted in 55\% sensitivity and $100 \%$ side concordance for detecting cervical spondylotic radiculopathy and can thus be considered as a useful, diagnostic adjunct in the electrophysiological assessment of spondylogenic myelopathy or radiculopathy. ${ }^{82}$

CSP also shows a high sensitivity for detecting spinal cord lesions, thus representing a highly sensitive and accurate diagnostic tool, but it is not used routinely for diagnosis of CSM. However, the measurement of the CSP can provide information beyond that obtained from the MEPs elicited by means of TMS. In fact, MEPs assess corticospinal tract dysfunction, often seen in CSM lesions as a consequence of disc protrusion and anterior cord indentation, whereas CSP, mediated by spinal reflexes with supraspinal descending control, may address a larger anatomical circuit or area comprising afferent, efferent and supraspinal inputs.

SEPs and MEPs may help in the assessment of disease severity and can be used for monitoring and quantifying sensory and motor function in the course of patient recovery. Indeed, these techniques provide an useful functional correlate for documenting pre- and postoperative neurological status. Recently, intraoperative monitoring was associated with better clinical outcomes and some increased hospital charges among discharges of simple spinal fusions and laminectomies in a large, multiyear data set. ${ }^{83}$ Monitoring of MEPs may be considered as an alternative to SEPs during anterior cervical procedures, but combined monitoring of MEPs and SEPs is always the ideal. Even if some results are encouraging, these electrophysiological techniques at present cannot be recommended as independent outcome predictors for CSM surgery. ${ }^{84}$ Importantly, the intraoperative monitoring in patients with spinal cord pathology can be only combined (MEPs+SEPs+EMG). The clinician should also consider intraoperative deltoid and biceps TES-induced MEPs and spontaneous EMG monitoring in order to reduce postoperative C5 nerve root palsy whenever there is potential for iatrogenic C5 nerve root injury.

Interestingly, in patients with MRI signs of spondylotic cervical cord compression but without clear clinical signs of myelopathy, risk of early progression into symptomatic CMS $(\leqslant 1$ year) was predicted by the presence of clinically symptomatic radiculopathy and abnormal SEPs and MEPs, whereas MRI signal abnormalities (hyperintensity) predicted the later $\left(>1\right.$ year) development of $\mathrm{CSM}^{85}$ In a recent study, CMCTs at the lower limbs, but not MRI-measured sagittal and parasagittal diameters of the spinal canal, were found to correlate with long-term functional outcome following surgical or conservative treatment. ${ }^{86}$

In conclusion, the studies reported in the present review illustrate that electrophysiological studies are very useful complementary investigations for assessing cervical cord dysfunction and have an important role in diagnosis and management of CSM.

\section{DATA ARCHIVING}

There were no data to deposit.

\section{CONFLICT OF INTEREST}

The authors declare no conflict of interest.

1 Brain WR, Northfield D, Wilkinson M. The neurological manifestations of cervical spondylosis. Brain 1952; 75: 187-225.

2 Lebl DR, Hughes A, Cammisa FP Jr, O'Leary PF. Cervical spondylotic myelopathy: pathophysiology, clinical presentation, and treatment. HSS J 2011; 7: 170-178.

3 Adams C. Cervical spondylotic radiculopathy and myelopathy. In: Vinken PJ, Bruyn GW (eds). Handbook of Clinical Neurology, Vol. 26: Injuries of the Spine and Spinal Cord, Part 2. North Holland: Amsterdam, 1976, pp 97-112.

4 Al-Mefty O, Harkey LH, Middleton TH, Smith RR, Fox JL. Myelopathic cervical spondylotic lesions demonstrated by magnetic resonance imaging. J Neurosurg 1988; 68: 217-222.

5 Nové-Josserand A, André-Obadia N, Mauguière F. Cervical spondylotic myelopathy: motor and somatosensory evoked potentials, clinical and radiological correlation. Rev Neurol (Paris) 2002; 158: 1191-1197.

6 Epstein NE, Epstein JA, Carras R. Coexisting cervical spondylotic myelopathy and bilateral carpal tunnel syndromes. J Spinal Disord 1989; 2: 36-42.

7 Bednarik J, Kadanka Z, Vohánka S. Median nerve mononeuropathy in spondylotic cervical myelopathy. J Neurol 1999; 246: 544-551.

8 Liu FJ, Sun YP, Shen Y, Du H. Prognostic value of magnetic resonance imaging combined with electromyography in the surgical management of cervical spondylotic myelopathy. Exp Ther Med 2013; 5: 1214-1218.

9 Liu M, Cui L, Guan Y, Li B, Du H. Single-fiber electromyography in amyotrophic lateral sclerosis and cervical spondylosis. Muscle Nerve 2013; 48: 137-139.

10 Malone A, Meldrum D, Gleeson J, Bolger C. Elecromyographic characteristics of gait impairment in cervical spondylotic myelopathy. Eur Spine J 2013; 22: 2538-2544.

11 Lo YL. How has electrophysiology changed the management of cervical spondylotic myelopathy? Eur J Neurol 2008; 15: 781-786.

12 Lo YL, Tan YE, Dan YF, Leoh TH, Tan SB, Tan CT et al. Cutaneous silent periods in the evaluation of cord compression in cervical spondylosis. J Neurol 2007; 254: 141-149.

13 Stetkarova I, Kofler M. Cutaneous silent periods in the assessment of mild cervical spondylotic myelopathy. Spine (Phila Pa 1976) 2009; 34: 34-42.

14 Kang DX, Fan DS. The electrophysiological study of differential diagnosis between amyotrophic lateral sclerosis and cervical spondylotic myelopathy. Electromyogr Clin Neurophysiol 1995; 35: 231-238.

15 El-Negamy E, Sedgwick EM. Delayed cervical somatosensory potentials in cervical spondylosis. J Neurol Neurosurg Psychiatry 1979; 42: 238-241.

16 Ganes T. Somatosensory conduction times and peripheral, cervical and cortical evoked potentials in patients with cervical spondylosis. J Neurol Neurosurg Psychiatry 1980; 43: 683-689.

17 Yu YL, Jones SJ. Somatosensory evoked potentials in cervical spondylosis. Correlation of median, ulnar and posterior tibial nerve responses with clinical and radiological findings. Brain 1985; 108: 273-300.

18 Veilleux M, Daube JR. The value of ulnar somatosensory evoked potentials (SEPs) in cervical myelopathy. Electroencephalogr Clin Neurophysiol 1987; 68: 415-423.

19 Mehalic TF, Pezzuti RT, Applebaum Bl. Magnetic resonance imaging and cervical spondylotic myelopathy. Neurosurgery 1990; 26: 217-226.

20 Restuccia D, Di Lazzaro V, Valeriani M, Tonali P, Mauguiere F. Segmental dysfunction of the cervical cord revealed by abnormalities of the spinal N13 potential in cervical spondylotic myelopathy. Neurology 1992; 42: 1054-1063.

21 Restuccia D, Di Lazzaro V, Lo Monaco M, Evoli A, Valeriani M, Tonali P. Somatosensory evoked potentials in the diagnosis of cervical spondylotic myelopathy. Electromyogr Clin Neurophysiol 1992; 32: 389-395.

22 Restuccia D, Valeriani M, Di Lazzaro V, Tonali P, Mauguière F. Somatosensory evoked potentials after multisegmental upper limb stimulation in diagnosis of cervical spondylotic myelopathy. J Neurol Neurosurg Psychiatry 1994; 57: 301-308.

23 Emerson RG, Pedley TA. Effect of cervical spinal cord lesions on early components of the median nerve somatosensory evoked potentials. Neurology 1986; 36: 20-26.

24 Urasaki E, Wada S, Kadoya C, Matsuzaki H, Yokota A, Matsuoka S. Absence of spinal N13-P13 and normal scalp far-field P14 in a patient with syringomyelia. Electroencephalogr Clin Neurophysiol 1988; 71: 400-404.

25 Restuccia D, Mauguiere F. The contribution of median nerve SEPs in the functional assessment of the cervical spinal cord syringomyelia. Brain 1991; 114: 361-379.

26 Mauguiere F, Restuccia D. Inadequacy of the forehead reference montage for- detecting abnormalities of the spinal N13 SEP in cervical cord lesions. Electroencephalogr Clin Neurophysiol 1991; 79: 448-456.

27 Ibafiez V, Fischer G, Mauguiere F. Dorsal horn and dorsal column dysfunction in intramedullary cervical cord tumours. A somatosensory evoked potential study. Brain 1992; 115: 1209-1234. 
28 Perlik SJ, Fisher MA. Somatosensory evoked response evaluation of cervical spondylitic myelopathy. Muscle Nerve 1987; 10: 481-489.

29 Mauguiere F, Ibafiez. The dissociation of early SEP components in lesions of the cervicomedullary junction: a cue for routine interpretation of abnormal cervical responses to median nerve stimulation. Electroencephalogr Clin Neurophysiol 1985; 62: 406-420.

30 Mauguiere F. Les potentiels evoques somesthesiques cervicaux chez le sujet normal: analyse des aspects obtenus selon le siege de l'electrode de' rference. Rev Electroencephogr Neurophysiol Clin 1983; 13: 259-272.

31 Garcia-Larrea Lo, Mauguiere F. Latency and amplitude abnormalities of the scalp far-field P14 to median nerve stimulation in multiple sclerosis. A SEP study of 122 patients recorded with a noncephalic reference montage. Electroencephalogr Clin Neurophysiol 1988; 71: 180-186.

32 Fujimoto H, Kaneko K, Taguchi T, Ofuji A, Yonemura H, Kawai S. Differential recording of upper and lower cervical N13 responses and their contribution to scalp recorded in median nerve somatosensory evoked potentials. J Neurol Sci 2001; 187: 17-26.

33 Dikmen PY, Oge AE. Diagnostic use of dermatomal somatosensory-evoked potentials in spinal disorders: Case series. J Spinal Cord Med 2013; 36: 672-678.

34 Abbruzzese G, Dall'Agata D, Morena M, Simonetti S, Spadavecchia L, Severi P et al. Electrical stimulation of the motor tracts in cervical spondylosis. J Neurol Neurosurg Psychiatry 1988; 51: 796-802.

35 Maertens de Noordhout MA, Remacle JM. Magnetic stimulation of the motor cortex in cervical spondylosis. Neurology 1991; 41: 75-80.

36 Di Lazzaro V, Restuccia D, Colosimo C, Tonali P. The contribution of magnetic stimulation of the motor cortex to the diagnosis of cervical spondylotic myelopathy. Correlation of central motor conduction to distal and proximal upper limb muscles with clinical and MRI findings. Electroencephalogr Clin Neurophysiol 1992; 85: 311-320.

37 Chan YC, Yeh IB, Kannan TA, Wilder-Smith E. Trapezius motor evoked potential in evaluations of corticospinal tract lesions. Eur J Neurol 2009; 16: 540-543.

38 Herdmann J, Dvorak J, Bock WJ. Motor evoked potentials in patients with spinal disorders: upper and lower motor neuron affection. Electromyogr Clin Neurophysiol 1992; 32: 323-330.

39 Tavy DLJ, Wagner GL, Keunen RWM, Wattendorff AR, Hekster RE, Franssen H. Transcranial magnetic stimulation in patients with cervical spondylotic myelopathy: clinical and radiological correlations. Muscle Nerve 1994; 17: 235-241.

40 Chistyakov AV, Soustiel JF, Hafner H, Feinsod M. Motor and somatosensory conduction in cervical myelopathy and radiculopathy. Spine 1995; 20: 2135-2140.

41 Bednarik J, Kadanka Z, Vohanka S, Novotný O, Surelová D, Filipovicová D et al. The value of somatosensory and motor evoked potentials in pre-clinical spondylotic cervical cord compression. Eur Spine J 1998; 7: 493-500.

42 Maertens de Noordhout AM, Myressiotis S, Delvaux V, Born JD, Delwaide PJ. Motor and somatosensory evoked potentials in cervical spondylotic myelopathy. Electroencephalogr Clin Neurophysiol 1998; 108: 24-31.

43 Tavy DL, Franssen H, Keunen RW, Wattendorff AR, Hekster RE, Van Huffelen AC. Motor and somatosensory evoked potentials in asymptomatic spondylotic cord compression. Muscle Nerve 1999; 22: 628-634.

44 Travlos A, Pant B, Eisen A. Transcranial magnetic stimulation for detection of preclinical cervical spondylotic myelopathy. Arch Phys Med Rehabil 1992; 73: 442-446.

45 De Mattei M, Paschero B, Sciarretta A, Davini O, Cocito D. Usefulness of motor evoked potentials in compressive myelopathy. Electromyogr Clin Neurophysiol 1993; 33: 205-216.

46 Kalupahana NS, Weerasinghe VS, Dangahadeniya U, Senanayake N. Abnormal parameters of magnetically motor evoked potentials in patients with cervical spondylotic myeloapthy. Spine J 2008; 8: 645-649.

47 Di Lazzaro V, Oliviero A. Evaluation of myelopathy, radikulopathy and thoracic nerve. In Hallett M, Chokroverty S (eds). Magnetic Stimulation in Clinical Neurophysiology. Butterworth-Heinemann: Philadelphia, PA, USA, 2005, pp 105-127.

48 Ugawa Y, Rothwell JC, Day BL, Thompson PD, Marsden CD. Percutaneous electrical stimulation of corticospinal pathways at the level of the pyramidal decussation in humans. Ann Neurol 1991; 29: 418-427.

49 Ugawa Y, Uesaka Y, Terao Y, Suzuki M, Sakai K, Hanajima R et al. Clinical utility of magnetic corticospinal tract stimulation at the foramen magnum level. Electroencephalogr Clin Neurophysiol 1996; 101: 247-254.

50 Deftereos SN, Kechagias EA, Panagopoulos G, Seretis A, Orphanidis G. Antoniou E et al. Localisation of cervical spinal cord compression by TMS and MRI. Funct Neurol 2009; 24: 99-105.

51 Funaba M, Kanchiku T, Imajo Y, Suzuki H, Yoshida Y, Nishida N et al. Transcranial magnetic stimulation in the diagnosis of cervical compressive myelopathy: comparison with spinal cord evoked potentials. Spine (Phila Pa 1976) 2015; 40: E161-E167.

52 Funaba M, Kanchiku T, Imajo Y, Suzuki H, Yoshida Y, Nishida N et al. Characteristics of C6-7 myelopathy: assessment of clinical symptoms and electrophysiological findings. Spinal Cord 2016; 54: 798-803.

53 Jaskolski DJ, Laing RJ, Jarratt JA, Jukubowski J. Pre- and postoperative motor conduction times, measured using magnetic stimulation, in patients with cervical spondylosis. Br J Neurosurg 1990; 4: 187-192.

54 Chang CW, Lin SM. Measurement of motor conduction in the thoracolumbar cord. Spine 1996: 21: 485-491.

55 Capone F, Tamburelli FC, Pilato F, Profice P, Ranieri F, Di lorio R et al. The role of motor-evokes potentials in the management of cervical spondylotic myelopathy. Spine J 2013; 13: 1077-1079.
56 Tanaka N, Nakanishi K, Fujimoto Y, Sasaki H, Kamei N, Hamasaki T et al. Clinical results of cervical myelopathy in patients older than 80 years of age: evaluation of spinal function with motor evoked potentials. J Neurosurg Spine 2009; 118 421-426.

57 Lyu RK, Tang LM, Chen CJ, Chen CM, Chang HS, Wu YR. The use of evoked potentials for clinical correlation and surgical outcome in cervical spondylotic myelopathy with intramedullary high signal intensity on MRI. J Neurol Neurosurg Psychiatry 2004; 75: 256-261.

58 Bednarik J, Kadank Z, Vohanka Z, Stelskal L, Vlach O, Schroder R. The value of somatosensory and motor evoked potentials in predicting and monitoring the effect of therapy in spondylotic cervical myelopathy. Prospective randomized study. Spine 1999; 24: 1593-1598.

59 Lo YL, Dan YF, Tan YE, Nurjannah S, Tan SB, Tan CT et al. Intraoperative motor evoked potential monitoring in scoliosis surgery: comparison of desflurane/nitrous oxide with propofol total intravenous anesthetic regimens. J Neurosurg Anesthesiol 2006; 18: 211-214.

60 Cui H, Wang Y, Li X, Xie X, Xu S, Hu Y. Trial-to-trial latency variability of somatosensory evoked potentials as a prognostic indicator for surgical management of cervical spondylotic myelopathy. J Neuroeng Rehabil 2015; 12: 49.

$61 \mathrm{Ma}$ Y, Hu Y, Valentin N, Geocadin RG, Thakor NV, Jia X. Time jitter of somatosensory evoked potentials in recovery from hypoxic-ischemic brain injury. J Neurosci Methods 2011; 201: 355-360.

62 Tani T, Ushida T, Yamamoto H. Surgical treatment guided by spinal cord evoked potentials for tetraparesis due to cervical spondylosis. Paraplegia 1995; 33 : 354-358.

63 Tani T, Ishida K, Ushida T, Yamamato H. Intraoperative electroneurography in the assessment of the level of operation for cervical spondylotic myelopathy in the elderly. J Bone Joint Surg B 2000; 82: 269-274.

64 Uchida K, Nakajima H, Sato R, Kokubo Y, Yayama T, Kobayashi S et al. Multivariate analysis of the neurological outcome of surgery for cervical compressive myelopathy. J Orthop Sci 2005; 10: 564-573.

65 Bouchard JA, Bohlman HH, Biro C. Intraoperative improvements of somatosensory evoked potentials: correlation to clinical outcome in surgery for cervical spondylotic myelopathy. Spine 1996; 21: 589-594.

66 Smith PN, Balzer JR, Khan MH, Davis RA, Crammond D, Welch WC et al. Intraoperative somatosensory evoked potential monitoring during anterior cervical discectomy and fusion in nonmyelopathic patients a review of 1039 cases. Spine J 2007; 7: 83-87.

67 Gokaslan ZL, Samudrala S, Deletis V, Wildrick DM, Cooper PR. Intraoperative monitoring of spinal cord function using motor evoked potentials via transcutaneous epidural electrode during anterior cervical spinal surgery. J Spinal Disord 1997; 10: 299-303.

68 Deletis V, Sala F. Intraoperative neurophysiological monitoring of the spinal cord during spinal cord and spine surgery: a review focus on the corticospinal tracts. Clin Neurophysiol 2008; 119: 248-264.

69 Sloan TB, Heyer EJ. Anesthesia for intraoperative neurophysiologic monitoring of the spinal cord. J Clin Neurophysiol 2002; 19: 430-443.

70 Deiner S. Highlights of anesthetic considerations for intraoperative neuromonitoring. Semin Cardiothorac Vasc Anesth 2010; 14: 51-53.

71 Lo YL, Dan YF, Tan YE, Nurjannah S, Tan SB, Tan CT et al. Intraoperative monitoring in scoliosis with multipulse cortical stimulation and desflurane anesthesia. Spinal Cord 2004: 42: 342-345.

72 Hillibrand AS, Schwartz DM, Sethuraman V, Vaccaro AR, Albert TJ. Comparison of transcranial electric motor and somatosensory evoked potential monitoring during cervical spine surgery. J Bone Joint Surg B 2004; 86 A: 1248-1253.

73 Clark AJ, Ziewacz JE, Safaee M, Lau D, Lyon R, Chou D et al. Intraoperative neuromonitoring with MEPs and prediction of postoperative neurological deficits in patients undergoing surgery for cervical and cervicothoracic myelopathy. Neurosurg Focus 2013; 35: E7.

74 Nakamae T, Tanaka N, Nakanishi K, Kamei N, Izumi B, Fujioka Y et al. Investigation of segmantal motor paralysis after cervical laminoplasty using intraoperative spinal cord monitoring with transcranial electrical motor-evoked potentials. J Spinal Disord Tech 2012; 25: 92-98.

75 Lee JY, Hillibarnd AS, Lim MR, Zavatsky J, Zeiller S, Schwartz DM et al. Characterization of neurophysiologic alerts during anterior spine surgery. Spine 2006; 31: 1916-1922.

76 Kaneko K, Hashiguchi A, Kato Y, Kojima T, Imajyo Y, Taguchi T. Investigation of motor dominant C5 paralysis after laminoplasty from the results of evoked spinal cord responses. J Spinal Disord Tech 2006; 19: 358-361.

77 Fan D, Schwartz DM, Vaccaro AR, Hillibarnd AS, Albert TJ. Intraoperative neurophysiologic detection of iatrogenic C5 nerve root injury during laminectomy for cervical compression myelopathy. Spine 2002; 27: 2499-2502.

78 Bose B, Sestokas AK, Schwartz DM. Neurophysiological detection of iatrogenic C5 nerve deficit during anterior cervical spinal surgery. J Neurosurg Spine 2007; 6: 381-385.

79 Resnick DK, Anderson PA, Kaiser MG, Groff MW, Heary RF, Holly LT et al. Joint section on disorders of the spine and peripheral nerves of the American Association of Neurological Surgeons and Congress of Neurological Surgeons. Ectrophysiological monitoring during surgery for cervical degenerative myelopathy and radiculopathy. $J$ Neurosurg Spine 2009; 11: 245-252.

80 Nardone R, Höller Y, Thomschewski A, Höller P, Bergmann J, Golaszewski S et al. Central motor conduction studies in patients with spinal cord disorders: a review. Spinal cord 2014; 52: 420-427. 
81 Di Lazzaro V, Oliviero A, Profice P, Ferrara L, Saturno E, Pilato F. The diagnostic value of motor evoked potentials. Clin Neurophysiol 1999; 110: 1297-1307.

82 Lo YL, Chan LL, Leoh T, Lim W, Tan SB, Tan CT et al. Diagnostic utility of F waves in cervical radiculopathy: electrophysiologcal and magnetic resonance imaging correlation. Clin Neurol Neurosurg 2008; 110: 58-61.

83 Ney JP, van der Goes DN, Nuwer MR. Does intraoperative neurophysiologic monitoring matter in noncomplex spine surgeries? Neurology 2015; 85: 2151-2158.
84 Jones SJ, Buonamassa S, Crockard HA. Two cases of quadriparesis following anterior cervical discectomy, with normal perioperative somatosensory evoked potentials. J Neurol Neurosurg Psychiatry 2003; 74: 273-276.

85 Bednarik J, Kadanka Z, Dusek L, Kerkovsky M, Vohanka S, Novotny 0 et al. Presymptomatic spondylotic cervical myelopathy: an updated predictive model. Eur Spine J 2008; 17: 421-431.

86 Deftereos SN, Kechagias E, loakeimidou C, Georgonikou D. Transcranial magnetic stimulation but not MRI predicts long-term clinical status in cervical spondylosis: a case series. Spinal Cord 2015; 53: S16-S18. 